\title{
Cerebral Arteriovenous Malformation Presenting as Acute Lacunar-Like Infarct Associated with Vascular Steal
}

\author{
Taewon Kim, MD, $\mathrm{PhD}^{*}$ (1); Seung-Jae Lee, $\mathrm{MD}, \mathrm{PhD}^{\dagger}$ (D) \\ Department of Neurology, Incheon St. Mary's Hospital, The Catholic University of Korea, College of Medicine ${ }^{*}$, Seoul; Department of \\ Neurology, Soonchunhyang University Bucheon Hospital ${ }^{\dagger}$, Bucheon, Korea
}

Cerebral infarction associated with cerebral arteriovenous malformation (AVM) is extremely rare. Herein, we describe a unique case of cerebral AVM presenting as acute lacunar-like infarction associated with vascular steal. A 46-year-old hypertensive man presented with a 2-day history of dysarthria and left hemiparesis of sudden onset. Diffusion-weighted magnetic resonance imaging (MRI) demonstrated a diffusion-restricted lesion in the right corona radiata. Therefore, he was initially diagnosed with lacunar infarction. However, subsequent computed tomographic angiography showed an AVM with calcification in the right corpus callosum and cingulate gyrus. Additional MRI sequences revealed chronic ischemic lesions exclusively in the right cerebral hemisphere. In addition, Technetium-99m-ethyl cysteinate dimer single-photon emission computed tomography showed diffuse hypoperfusion in the right cerebral hemisphere. Thus, hypoperfusion associated with vascular steal could have contributed to the development of the lesion, although our patient might have been predisposed to "lacunar" infarct due to hypertension.

J Neurosonol Neuroimag 2020;12(2): 6 6-7S

Key Words: Cerebral infarction; Arteriovenous malformations; Hemodynamics; Steal
Received: October 6, 2020

Revised: November 10, 2020

Accepted: November 20, 2020

Correspondence:

Seung-Jae Lee, MD, $\mathrm{PhD}$

Department of Neurology, Soonchunhyang University Bucheon Hospital, 170 Jomaru-ro, Wonmi-gu, Bucheon 14584,

Korea

Tel: $+82-32-621-6548$

Fax: $+82-32-621-6950$

E-mail:neurosj@catholic.ac.kr
Cerebral arteriovenous malformation (AVM) is a disease characterized by a complex tangled web of abnormal vascular connections between the arterial feeders and the draining veins. ${ }^{1}$ AVMs are associated with high morbidity and mortality, mainly due to hemorrhagic complications.

Vascular steal is a complicated phenomenon that is associated with cerebral AVM. ${ }^{2}$ However, vascular steal in cerebral AVM very rarely causes ischemic stroke, and only a few such cases have been reported. ${ }^{3}$ Here, we describe a unique case of cerebral AVM presenting as acute lacunar-like infarction, presumably resulting from vascular steal.

\section{CASE}

A 46-year-old hypertensive, but otherwise healthy, man presented with a 2-day history of dysarthria and left hemiparesis of sudden onset. On admission, he was not on antihypertensive medication, and his blood pressure was 157/128 mmHg. Initial neurological examination showed dysarthria, left central-type facial palsy, and left hemiparesis (Medical Research Council [MRC] motor grade IV). Diffusion-weighted magnetic resonance imaging (MRI) revealed an acute, small, solitary infarct in the right corona radiata (Fig. 1A). Therefore, he was initially diagnosed with acute lacunar infarction, and was treated with $100 \mathrm{mg}$ aspirin and $40 \mathrm{mg}$ atorvastatin. Subsequently, head-and-neck computed tomographic angiography revealed normal vessels, apart 
from an AVM with calcification in the right corpus callosum and cingulate gyrus (Fig. 1B, C). Additional MRI sequences were acquired, which showed multiple flow voids corresponding to the AVM (Fig. 1D, arrow), and revealed chronic white matter hyperintensities and multiple chronic lacunar infarcts with mild cortical
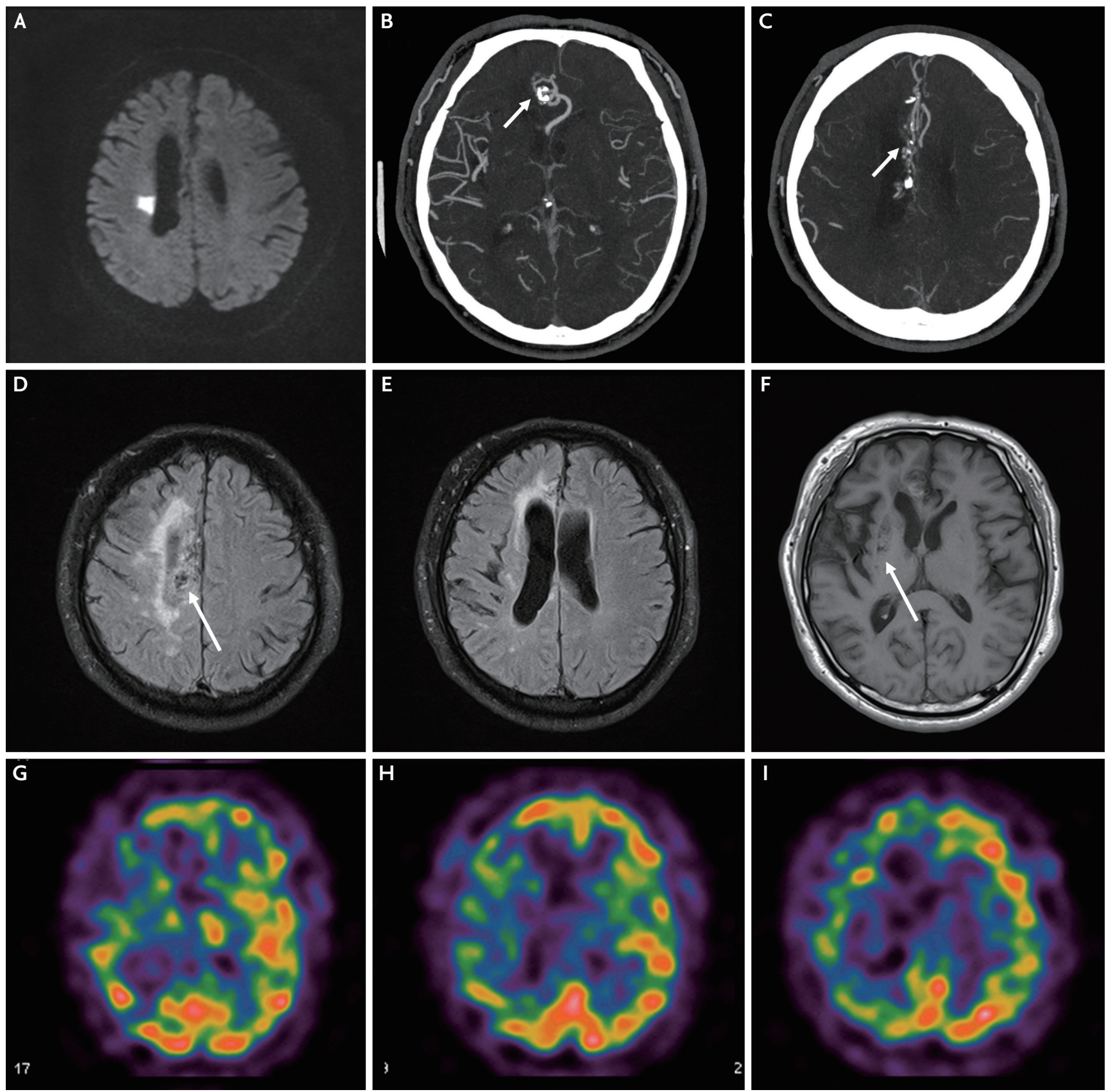

FIG. 1. (A) Diffusion-weighted magnetic resonance imaging (MRI) demonstrates a diffusion-restricted lesion in the right corona radiata, suggesting acute infarction. (B, C) Computed tomographic angiography shows an arteriovenous malformation (AVM) located in right corpus callosum and cingulate gyrus, which has the right anterior cerebral artery as a feeder artery (arrows). (D, E) Fluid-attenuated inversion recovery MRIs reveal multiple flow voids corresponding to the AVM (arrow of D), and periventricular white matter hyperintensities involving the right cerebral hemisphere but sparing the left cerebral hemisphere. (F) Tr-weighted MRI shows mild cortical atrophy, ventricular dilatation, and chronic multiple lacunar infarcts (arrow) exclusively in the right cerebral hemisphere, with no definite ischemic lesions in the left cerebral hemisphere. (G-I) The technetium-99m-ethyl cysteinate dimer single-photon emission computed tomography demonstrates hypoperfusion in the right cerebral hemisphere. 
atrophy and ventricular dilatation in the right cerebral hemisphere (Fig. 1E, F). However, there were no definite ischemic lesions in the left cerebral hemisphere. Technetium-99m-ethyl cysteinate dimer single-photon emission computed tomography showed diffuse hypoperfusion in the right cerebral hemisphere (Fig. 1GI).

He underwent heart examinations, including transthoracic echocardiography and 24-hour Holter monitoring, all of which had normal results. His clinical course during admission was stable with aspirin and statin treatment. However, there was not much improvement in his hemiparesis.

He refused further investigations, such as catheter angiography, and was discharged from the hospital on the 7th day. At the 6-month follow-up, he still had left hemiparesis (MRC motor grade IV) with a modified Rankin scale score of 2 .

\section{DISCUSSION}

Cerebral AVM usually manifests as hemorrhage or seizure. ${ }^{1}$ Focal neurological deficits due to cerebral infarction are quite rare $(<0.1 \%) .^{4}$ Previous studies have reported large artery territory infarcts associated with AVM. ${ }^{3-5}$ Unlike the previous studies, the present case uniquely manifested as an acute lacunar-like, that is, a small, solitary infarct.

Cerebral infarction associated with AVM may result from various etiologies, including coincidental cardioembolic or atherothrombotic lesions, ${ }^{4,5}$ steno-occlusion of a feeding artery due to fibromuscular proliferation or endothelial injury associated with turbulent high velocity blood flow, ${ }^{4,6,7}$ or vascular steal, which diverts blood flow from adjacent brain tissue into the low resistance network of the AVM. ${ }^{3}$ Although hypertension might have predisposed our patient to the "lacunar" infarct in this case, hypoperfusion associated with vascular steal could have contributed much more to the development of the lesion, given the imaging findings of chronic ischemia exclusively involving the right hemisphere around the AVM with sparing of the left hemisphere.

\section{Acknowledgements}

This study was supported by the Soonchunhyang University Research Fund.

\section{Conflicts of Interest}

No potential conflicts of interest relevant to this article was reported.

\section{REFERENCES}

1. Solomon RA, Connolly ES Jr. Arteriovenous malformations of the brain. N Engl J Med. 2017;376:1859-1866.

2. Marks MP, Lane B, Steinberg G, Chang P. Vascular characteristics of intracerebral arteriovenous malformations in patients with clinical steal. AJNR Am J Neuroradiol. 1991;12:489-496.

3. Ellis MJ, Armstrong D, Dirks PB. Large vascular malformation in a child presenting with vascular steal phenomenon managed with pial synangiosis. J Neurosurg Pediatr. 2011;7:1521.

4. Goto H, Suzuki M, Akimura T, Fujisawa H, Yoneda H, Oka F, et al. Progression of stenosis into occlusion of the distal posterior cerebral artery supplying an occipital arteriovenous malformation manifesting as multiple ischemic attacks: case report. Neurol Med Chir (Tokyo). 2012;52:899-902.

5. Komiyama M, Nakajima H, Nishikawa M, Yasui T. Embolic stroke in the territory of a cerebellar arteriovenous malformation--case report. Neurol Med Chir (Tokyo). 1998;38:417419.

6. Enam SA, Malik GM. Association of cerebral arteriovenous malformations and spontaneous occlusion of major feeding arteries: clinical and therapeutic implications. Neurosurgery. 1999;45:1105-1111; discussion 1111-1112.

7. Mawad ME, Hilal SK, Michelsen WJ, Stein B, Ganti SR. Occlusive vascular disease associated with cerebral arteriovenous malformations. Radiology. 1984;153:401-408. 